\title{
PLOTINO: PROVIDENCIA Y PARTICIPACIÓN EN EL COSMOS SENSIBLE. SU PROYECCIÓN EN LA HIPÓTESIS GAIA
}

Plotinum: Providende and Participation in the sensitive cosmos. Your projection in The Gaia hypothesis

Marcelo Andrés Poblete ${ }^{1}$

\begin{abstract}
RESUMEN
Nuestro escrito tiene por finalidad, por una parte; exponer categorías y doctrinas de orden metafísico que fundamentan la cosmología del pensamiento plotiniana. Algunas de esas nociones filosóficas son; Doble logos, Providencia, Contemplación, Mundo Sensible y Mundo Inteligible. Por otro lado, queremos describir de modo general la concepción de la Tierra como megaorganismo vivo autorregulable propuesto por el científico británico; James Lovelock.Y, por último, establecer una comparación entre ambos enfoques para de allí derivar diferencias y posibles semejanzas.
\end{abstract}

Palabras claves: Neoplatonismo. Cosmología plotiniana. Providencia. Cosmos sensible. Lovelock. Hipótesis Gaia.

\begin{abstract}
Our writing is intended, on the one hand; expose categories and doctrines of a metaphysical order that the basis for the cosmology of Plotinian thought. Some of those philosophical notions are; Double logos, Providence, Contemplation, Sensible World and Intelligible World. On the other hand, we want to describe in a general way the conception of the Earth as a self-regulating living mega-organism prosed by the British scientist; James Lovelock. And, finally, establish a comparison between both approaches and possible similarities from there.
\end{abstract}

Keywords: Neoplatonism. Plotinian cosmology. Providence. Sensitive cosmos. Lovelock. Gaia hypothesis.

\section{Introducción}

El presente trabajo tiene como objetivo principal exponer brevemente las nociones metafísicas afines a la Doctrina de la Providencia, en el pen-

\footnotetext{
${ }^{1}$ Departamento de Filosofía y Ciencias de la Educación de la Facultad de Filosofía, Humanidades y Artes de la Universidad Nacional de San Juan. E-mail: mpoblete@hotmail.com . ORCID: https://orcid.org/0000-0002-6435-8808.
} 
samiento plotiniano. En razón de ello, y en primer lugar, nos interesará rescatar las importantes consecuencias cosmológicas que se infieren como resultado de la relación Providencia, logos, Contemplación y Mundo Sensible, en la obra de Plotino. Puesto que en su sistema místico-metafísico dichas nociones contribuyen a consolidar la defensa de la dignidad del cosmos y a refrendar la posición cosmista sostenida por nuestro pensador. Un punto interesantísimo para destacar en favor de este cosmismo es el hecho de que la Providencia o pro/noia garantiza la huella del Uno en todos y cada uno de los seres vivos, avalando de este modo, la capacidad de contemplación y la de participación en la Unidad.

En segundo lugar, diremos que Plotino considera que la Participación del Cosmos Sensible se manifiesta en el hecho de que éste último es imagen del Cosmos Inteligible, o Modelo, que se encuentra en la Hipóstasis Inteligencia, dentro de su sistema de las tres hipóstasis.

Para ampliar la relación Providencia-Participación-Cosmos Sensible nombraremos las siguientes líneas de acción bajo las que se desarrollará y reflejará la Doctrina de la Participación del cosmos sensible, phýsis o Naturaleza, en la Unidad. A saber: 1) Providencia, Participación y Doble Logos; 2) Providencia y Contemplación en la phýsis (físis); 3) Alma del Cosmos y Participación; 4) Propiedades del Alma del Cosmos; 5) Su proyección en la hipótesis Gaia; 6) Aspectos fundamentales de la Hipótesis Gaia y 7) Comparación de la Proyección: entre Hipótesis Gaia y la cosmología plotiniana.

\section{1- Providencia, Participación, y Doble Logos}

Ahora bien, es necesario delimitar el significado que adquiere la noción de Providencia en Plotino para comprender el título que le da nombre al presente trabajo. Dicha noción debe ser concebida como una actividad de la Hipóstasis Alma entendida como $\operatorname{logos},{ }^{2}$ cuya primera función consiste en

2 Para comprender la organización y correspondiente jerarquización recurrimos al traductor español de la Enedas, Jesús Igal, quien afirma que: “[...] el responsable último de la Providencia es el Uno-Bien, que la garantiza con su sola existencia. Pero, estrictamente hablando, aun la Inteligencia está más allá de la Providencia, que propiamente es tarea del Alma entendida como Logos. Pero como hay dos niveles de Alma y, correspondientemente, dos Logos, así hay también dos niveles de Providencia. La Providencia total o perfecta consta de Providencia superior, que es un Logos «empalmador» del mundo inteligible con el sensible, y de Providencia inferior, que es un Logos «empalmado» con el anterior y «ejecutor» del orden cósmico". Introducción General, secc. 63, punto 2. 
que este cosmos sensible esté en conformidad con la Hipóstasis Inteligencia. ${ }^{3} \mathrm{Su}$ manifestación se vislumbra en el hecho de que todas y cada una de las cosas de nuestro mundo estén correctamente dispuestas, y que el conjunto de todas ellas esté prolijamente coordinado.

Por otro lado, la Providencia total o perfecta es el resultado de una división en dos niveles, a saber: una Providencia superior y otra inferior. La primera, al nivel de la Inteligencia, contempladora de la Hipóstasis Inteligencia y las Formas (morphé) ${ }^{4}$, encargada de la conformidad del mundo sensible con el inteligible; la segunda, ubicada al nivel del Alma Inferior o Alma del cosmos, inmanente y trascendente a la fu/sij. Es importante recordar que la Providencia solo interviene mediante la acción del logos. Esa intervención de la prónoia en el mundo sensible es la expresión evidente de la Doctrina de la Participación mediante la actividad llevada a cabo por la Doctrina del Doble Logos que es un requisito indispensable para la ejecución de la presencia de la Providencia en el mundo sensible. En efecto, ésta es la manera en que la Providencia se manifiesta en el Cosmos Sensible mediante la intervención del segundo logos, logos ejecutor o logos proódico.

Es por ello que la definición de Providencia que hemos obtenido y su relación con la Doctrina del Doble Logos, alcanza su real y verdadera dimensión cuando se la vincula a la capacidad de contemplación que posee la Naturaleza, tema que será desarrollado a continuación.

\section{2- Providencia y Contemplación en la phýsis}

Para sostener y fundamentar la universalización de la Contemplación a todos los seres de la phýsis, será de suma utilidad el análisis de algunos fragmentos del sugerente tratado que lleva por título Sobre la Naturaleza, la contemplación y el uno, (III 8 [30]). Dicho estudio redundará en la interpretación acerca de una Naturaleza contemplativa, como así también servirá para establecer relaciones y comparaciones entre la Doctrina de la Providencia y la Doctrina de la Contemplación, ambas presentes en la phýsis.

\footnotetext{
${ }^{3}$ Cf. En. III 2 [47] 1, 22-23: “[...] para el universo, la providencia consiste en que el cosmos esté en conformidad con la Inteligencia [...]".

4 Plotino usa el término griego morphé, és (para expresar las formas que advienen a la materia.
} 
Para introducirnos en la temática en cuestión, recordaremos el pasaje en que Plotino describe el alcance que tiene la Providencia:

¿Es que la providencia no se extiende hasta la tierra?

- Bien al contrario, puesto que las demás cosas suceden en virtud de un plan racional, ello prueba que la providencia se extiende también hasta la tierra. Pues aun los animales y las plantas participan de razón, de alma y de vida. (En. III 2 [47], 7, 33-37)

Consideramos que los aspectos notables de este fragmento en cuanto a la relación Providencia y Contemplación son los siguientes:

a) La Providencia se halla extendida en este mundo hasta los seres más ínfimos. b) En consecuencia, la Doctrina de la Contemplación se universaliza a todo ser vivo, y por lo tanto, se extiende la participación a todos ellos, como luego se verá. c) Un punto no menos importante es que si la Providencia se encuentra presente en todos los seres del planeta, ello no obstruye el ejercicio de la libertad individual, puesto que en nuestras acciones la libertad implica responsabilidad. Esta idea Plotino la toma de Platón: "la responsabilidad es de quien hizo la elección" (Republica, $617 e$ $4)^{6}$.

En cuanto al ítem $a$, diremos que para Plotino se trata de una firme convicción y un supuesto del cual él parte para organizar todo su sistema. Cada ser de la Naturaleza anhela indefectiblemente la Unidad, aunque existan partes en conflicto al interior del conjunto. Precisamente esta afirmación sostiene y fundamenta lo dicho en el punto $b$, es decir la universalización de la Contemplación a todos los seres de la phýsis. El siguiente pasaje de III 8 contribuirá a la interpretación acerca de una Naturaleza contemplativa, en el que en un tono de "aparente broma", el filósofo neoplatónico explica que:

Si comenzáramos bromeando antes de ponernos a hablar en serio y dijéramos que todos los seres aspiran a la contemplación y que éste es el fin al que miran no sólo los animales racionales, sino aun los irracionales y la Naturaleza que reside en las plantas y la tierra que las cría y que todos los seres la alcanzan en la medida en que les es posible mientras se hallan en su estado natural, pero que unos contemplan y alcanzan la contemplación de una manera y otros de

\footnotetext{
5 La idea de que la Providencia está expandida y que, por lo tanto, alcanza a toda la Tierra se reitera en otras oportunidades: En. III 2 [47], 6, 20-26; 13, 17-28; En. III 3 [48], 6, 8-13.

6 En. III 2 [47] 7, 20.
} 
otra, unos de verdad y otros percibiendo una imitación e imagen ${ }^{7}$ de ella, ¿habría alguien que tolerara lo paradójico de esta afirmación? (En. III 8 [30] 1, 1-9.)

En primer lugar, el pasaje muestra de modo claro que ambas Doctrinas, Providencia y Contemplación, están estrechamente conectadas y de alguna manera representan la característica trascendente de la Providencia y, por lo tanto, la Participación de la Naturaleza en el logos. En efecto, la capacidad de generación de la phýsis se halla en su actividad contemplativa. Pero también, el fragmento citado argumenta en favor de la contemplación de todos los seres según sus posibilidades; unos de manera directa o plena, y otros de manera indirecta; mediante una imitación.

Debido a que la temática que nos ocupa (Providencia-ParticipaciónCosmos Sensible) se enmarca en el terreno de la cosmología. Por ello, consideramos de gran interés que en el siguiente detenernos en el análisis de las funciones de la antiquísima noción del Alma cósmica.

\section{3- Plotino: tradición neoplatónica, Alma del cosmos y Participación}

La noción de Alma del cosmos es parte de la cosmología que se encuentra elaborada en la obra del Timeo, de Platón. Precisamente la noción de Alma del cosmos es concebida como la causa integradora y organizadora del mundo, que permite que éste sea un todo ordenado o Cosmos, portador de un equilibrio dinámico.

Plotino inserta al Alma del cosmos en el nivel inferior de la Hipóstasis $^{8}$ Alma $^{9}$, tercer rango ontológico perteneciente a su sistema místico-metafísico. Esta tercera Hipóstasis se configura en función de la doctrina

\footnotetext{
${ }^{7}$ Los términos mimesis e eikóna son traducidos como imitación e imagen, respectivamente.

${ }^{8}$ Por Hipóstasis debe entenderse un nivel o plano ontológico de la realidad, en otras palabras; un orden de realidad existente, pero de dimensión inteligible. Nos parece importante aportar la definición de hipóstasis encontrada: "«Hipóstasis» significa, a un tiempo, a) existencia autónoma de algo; b) su estructura formal; c) su posición en un orden” Infra 9, aclarado por ARANA MARCOS, 1998, p. 593. Pero más precisa pareciera ser la explicación aportada por F. García Bazán para el uso que del término efectúa Plotino: "La realidad, entonces, como queda claro, es experimentada o vivenciada por Plotino en tres niveles de profundidad que subsisten por sím mismos: se trata de realidades no ilusorias sino permanentes, y que subyacen bajo los seres perceptibles, impermanentes y cambiantes, por eso son hipos-

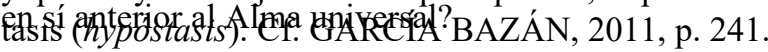


platónica del Alma cósmica, desarrollada por Platón en el Timeo. Pero esta doctrina es transformada profundamente por Plotino interpretándola, por una parte, en términos de los tres niveles psíquicos del alma humana de Aristóteles y adaptándola, por otra parte, a las leyes de su propia teoría de la procesión. Recordemos las palabras de Platón cuando expresa que: “... el cosmos es un animal que consta de cuerpo, alma e inteligencia, la inteligencia en el alma y el alma en el cuerpo" (Timeo, 30b). En esos pasajes del Timeo, Plotino parece descubrir los tres niveles psíquicos del alma humana de Aristóteles: el intelectivo, el sensitivo y el vegetativo. Por otra parte, estos tres niveles son reductibles a dos: el intelectivo, que constituye el Alma superior, y el sensitivo-vegetativo, que constituye el Alma inferior (V 2 [11] 1, 19-21). El Alma superior se identifica con la esencia indivisa del Timeo, y la inferior, a su vez, con la que se divide en los cuerpos (IV 3 [27] 19). El nivel superior de la tercera hipóstasis corresponde al Alma trascendente, separada del cuerpo. Mientras que el nivel inferior de dicha hipóstasis corresponde al Alma inmanente, ligada al cuerpo y mezclada con él (IV 3 [27] 14). Por la primera, el Alma se mantiene en contacto con el mundo inteligible y mediante la segunda, con el sensible. De este modo se expresaría la Participación en la cosmología de nuestro pensador.

Es importante atender a la actividad ejercida por sendos niveles de la tercera hipóstasis porque de ella dependerá la generación de este Cosmos Sensible. La actividad del Alma superior no es puramente contemplativa, mientras que la de la inferior o Physis tampoco es puramente creativa. Por ello Jesús Igal afirma que “[...] Contemplación y creación son dos aspectos de una misma actividad, dos facetas de un mismo Logos" (Introducción General a la traducción española, secc. 41, p. 57.). La actividad contemplativo-creativa del Alma consta, eso sí, de dos niveles: uno en el Alma superior y otro a nivel del Alma inferior, pero no de dos fases, puesto que en ambos niveles la contemplación y la creación son un solo acto, ni de dos procesos, ya que en ambos niveles ese único acto contemplativo-creativo es un acto indiviso e intemporal, por más que los productos resultantes estén

-Sí, hay que llamar Alma en sí a la que existe en la Inteligencia antes de que nazca el Alma". En. V 9 [5] 13, 20-23. 
sujetos a las coordenadas espacio-tiempo. Eso es lo que ocurre cuando Plotino explica cómo se generó el Cosmos sensible:

Así pues, puesto que lo originado es el cosmos entero, si te fijaras en él, tal vez escucharías de él estas palabras: «A mí me ha creado un dios y de él nací yo perfecto, integrado por todos los vivientes, contento conmigo mismo y autosuficiente, pues no necesito de nada. Porque en mí están todas las plantas, todos los animales y todos los seres originados por naturaleza, muchedumbre de dioses, pueblos de démones, almas buenas y hombres dichosos por virtud. Pues no es cierto que la tierra sí esté adornada de todas las plantas y de animales de todas clases y que el poder del Alma haya llegado hasta el mar, pero que el aire todo, el éter y el cielo enteros no tengan participación en el Alma... (En. III 2 (47) 3, 20-28.)

En el pasaje puede apreciarse que la Naturaleza en tanto Logos que es, también es una fuerza vital y creadora que crea espontáneamente siguiendo unas «órdenes» y esas órdenes son sin dudas los logoi recibidos del Alma superior.

Como acabamos de ver Plotino introduce movimiento y dinamismo en las correspondientes Hipóstasis, más específicamente a nivel del Alma del cosmos. Allí puede apreciarse que a ese despliegue del Logos se le añade el desarrollo de la noción platónica de participación con la que mitiga la idea de un mundo estrictamente sensible propuesta por Platón. Plotino explica que;

[...] Por otra parte, aducirán el pasaje en que también Platón expresa esta opinión cuando, al probar que el universo es un ser animado, dice que así como nuestro cuerpo es una parte del universo, así también nuestra alma es una parte del Alma del cosmos. Añadirán que se dice y se demuestra claramente que nosotros somos acompañantes de la rotación del universo y que, puesto que recibimos nuestros caracteres y nuestras suertes del universo y nacemos dentro de él, recibimos nuestra alma del medio que nos rodea; y que del mismo modo que, en nosotros, cada parte nuestra participa de nuestra alma, así también en la misma proporción nosotros, que somos partes con respecto al todo, participamos del Alma total a modo de parte (En. VI 3 [27] 1, 23-34). ${ }^{10}$

Como puede apreciarse en el fragmento, el filósofo neoplatónico explica la noción de participación sugiriéndose que las almas individuales for-

\footnotetext{
10 Negritas nuestras. Es preciso transcribir la explicación que Jesús Igal nos brinda acerca de cómo entienden los estoicos y Plotino el término «partes»: "Aquí radica la diferencia es encial entre los estoicos y Plotino: [...] para los primeros, el alma es cuerpo y las almas particulares son «fragmentos» de un mismo cuerpo, mientras que, para Plotino, el alma es incorpórea y las almas particulares son partes idénticas al todo". Infra 21, Enéada IV, p. 316.
} 
man parte de la del universo, aspecto esencial para comprender nuestro planteo.

A continuación, repasaremos algunas propiedades del Alma cósmica que, simultáneamente, posicionan a Plotino como un pensador defensor de la dignidad del Cosmos.

\section{4- Propiedades del Alma del cosmos}

Dado que el Alma inferior, el Alma del cosmos o la physis procede de la Hipóstasis Alma, reúne propiedades de la región inteligible que se manifiestan en el cosmos sensible. Entre esas propiedades las que debemos destacar son las del gobierno ${ }^{11}$ del mundo sensible, así como también la inserción del orden entre los seres que en él habitan. Otras cualidades del Alma del cosmos que no podrán pasar inadvertidas y que debemos mencionar es su eternidad y la posibilidad de relacionarse con las almas individuales $^{12}$.También diremos que el Alma inferior es única ${ }^{13}$, es sabiduría plena ${ }^{14}$,

\footnotetext{
11 “.... Porque el Alma, al tomar otra característica además de la de ser intelectiva, no se quedó en mera inteligencia. Y así, también el Alma posee su función, puesto que todo cuanto pertenece al reino de los inteligibles la posee. Mas el Alma, al mirar a quien es anterior a ella, intelige; pero, al mirarse a sí misma, pone en orden y gobierna al que es posterior a ella, y manda en él pues ni siquiera era posible que todas las cosas se detuviesen en la inteligible (panta en $t \% \frac{1}{2} 2$ noht $\%$ ) cuando era posible que, a continuación, viniese también a la existencia otro que, aunque es inferior, tenía que existir necesariamente, puesto que también existe necesariamente quien es anterior a él" (En. IV 8 [6] 3, 24-30).

12 "Por eso dice (Platón) que también nuestra alma, si se junta con aquella, que es perfecta, hecha perfecta ella misma «viaja por las alturas y gobierna todo el cosmos»; o sea que, cuando se mantenga aparte para no estar dentro de los cuerpos ni ser alma de un cuerpo particular, entonces también ella, como la del universo cogobernará el universo con facilidad [...]". En. IV 8 [6] 2, 20-24.

13 "Sin embargo, basándose en hechos de signo contrario, la razón dice que los hombres simpatizamos unos con otros cuando, al vernos, nos condolemos y nos sentimos relajados y naturalmente atraídos a la amistad. ¿No será que la amistad se debe a esto? Y si los encantamientos y las artes mágicas, en general, logran que nos juntemos y simpaticemos desde lejos, eso es posible sin duda por la unidad del alma y una palabra, en fin, dicha en voz baja influye en la disposición de quien está lejos y se hace escuchar por quien se halla a distancias inmensas. De todo lo cual cabe deducir que la unidad de todas las cosas se debe a que el alma es una sola" En. IV 9 [8] 3, 1-10.

14 “-Si, pues, nos asemejamos por la virtud, ¿nos asemejamos a quien posee virtud? Concretamente, ¿a qué Dios nos asemejamos? ¡Al que mejor parece poseer estas virtudes, más concretamente, al Alma del cosmos y al principio rector que hay en ella, dotado de una sabiduría maravillosa? Es, en efecto, razonable que sea él a quien tratemos de asemejarnos mientras estábamos acá" En. I 2 [19] 1, 6-9. Igal aclara que el principio rector que menciona Plotino debe ser entendido como la inteligencia propia del Alma del cosmos. Cf. nota 2 en Enéada I 2 [19].
} 
que se encarga del cuidado de los seres sensibles ${ }^{15}$. Sin embargo, habrá que recordar que fundamentalmente ella se encarga de ordenar la vida de la multiplicidad de los seres del cosmos sensible. Estas propiedades resaltan el desempeño destacado de esta valiosa noción dentro del sistema procesional de las tres hipóstasis.

\section{5- Ecofilosofía: Plotino y las bases filosóficas y metafísicas en la nueva Ecología}

En virtud de todo lo expuesto hasta aquí podemos afirmar que Plotino logra la universalización de la Contemplación, y su consecuente extensión a la totalidad de la Naturaleza, y por ende la participación de toda ella en el Uno. Nuestra tarea no ha sido corroborar dicha universalización a través de experiencias realizadas directamente en la Naturaleza, sino que se ha concretado en el análisis que Plotino hizo de algunos términos y principios metafísicos claves, que han permitido constituir las bases filosóficas de la ecología contemporánea. Uno de esos principios plotinianos es la armónica relación que el hombre debe mantener con el Cosmos. Otro principio en favor de la participación es que para Plotino, el Cosmos Sensible no puede existir sin un estrecho vínculo con su Modelo. Sin duda, esa compleja relación entre hombre y Cosmos es un tema fundamental en el amplio debate de la ecología. Por esta razón, estamos convencidos de que el análisis tanto de la doctrina de la Providencia, como la doctrina de la Participación y el Paradigma del Alma cósmica en el pensamiento del maestro neoplatónico, permitirá comprender la dignidad ontológica que le concede al Cosmos, proyectándolo en las bases epistemológicas de la ecología y ubicando a nuestro filósofo como un precursor de la disciplina que hoy conocemos con el nombre de Ecología. En razón de todo lo afirmado anteriormente expondremos una línea de investigación, dentro de las denominadas Ciencias de la Tierra, reconocida como la Hipótesis Gaia. Sostendremos que dicha Hipótesis mantiene ciertas semejanzas con la concepción metafísica plotiniana del alma cósmica o Cosmos sensible.

\footnotetext{
15 “[...] El cuidado de toda cosa es, efectivamente, de dos clases: uno universal, mediante órdenes de quien descansadamente pone orden con soberanía regia, y otro particular, actuando ya a las inmediatas por sí mismo, con lo que el agente, por su contacto con la cosa que se realiza, se contamina con la naturaleza del producto" En. IV 8 [6] 2, 27-30.
} 


\section{6- Hipótesis Gaia: aspectos fundamentales de la teoría}

En 1954, fue Hutchinson quien afirmó que el parentesco entre la materia viva y los productos de su descomposición debían convertirse en un tema central de investigación. Luego, James Lovelock, científico inglés, desde 1969, abordará la cuestión de la Tierra comprendiendo que ella se comporta como un gran organismo vivo. Contrariamente a la composición de las atmósferas de Marte y Venus, planetas sin vida, que pueden ser incluidos perfectamente en el marco de las leyes de la física y la química, la singularidad de la atmósfera terrestre puede tener una sola explicación: la actividad bioquímica de los seres vivos. En particular, el 21\% de oxígeno de la atmósfera terrestre es el resultado de la actividad fotosintética de los vegetales; pero este gas es tan reactivo que habría desaparecido, a escala de las eras geológicas, si no hubiera sido renovado por los procesos de la vida.

Además, se presentaba otro factor importante a tener en cuenta. El hecho de que para comprender la historia de la biosfera Lovelock sugeriría poner el acento en otra división que no sea la diferenciación clásica entre los dos reinos, animal y vegetal. Esta otra división, no menos fundamental, separa los procariotas, es decir, las bacterias, organismos constituidos de células sin núcleos, y las eucariotas, es decir, las otras formas vivas. Según Lovelock y Margulis, la superficie de la Tierra y su temperatura han estado continuamente reguladas desde que las procariotas aparecieron y colonizaron el planeta. A partir de sus formas más antiguas y más primitivas, la biota habría pues controlado activamente la composición química de la atmósfera y la temperatura, de forma que se mantuvieran las condiciones físico-químicas de su propia existencia. Así, durante los dos primeros miles de millones de años de la vida terrestre, las procariotas transformaron profundamente la superficie y la atmósfera terrestres, jugando el papel de termostato planet rio. El prolongado proceso de autorregulación ${ }^{16}$ habría sido como se describe

16 El científico británico afirma que; "Utilizo a menudo la palabra Gaia como abreviatura de la hipótesis misma, a saber: la biosfera es una entidad autorregulada con capacidad para mantener la salud de nuestro planeta mediante el control el entorno químico y el físico. Ha sido ocasionalmente difícil, sin acudir a circunlocuciones excesivas evitar hablar de Gaia como si fuera un ser consciente: deseo subrayar que ello no va más allá del grado de personalización que a un navío le confiere su nombre, reconocimiento a fin de cuentas de la identidad que hasta una serie de piezas de madera y metal puede ostentar cuando han sido específicamente diseñadas y ensambladas, del carácter que trasciende a la simple suma de las partes" (LOVELOCK, 2013, p. 8). [Versión Word en castellano GAIA; una nueva vi- 
a continuación.

Se sabe que el planeta Tierra es, entre todos los cuerpos celestes conocidos, absolutamente singular. Su posición frente al Sol, el equilibrio de las fuerzas gravitacionales y electromagnéticas, y la gran abundancia de agua en estado líquido llevaron a una plétora de especies de moléculas. Decisivo fue, entonces, el momento en que ocurrió la diversificación de las moléculas orgánicas. Ya los átomos de carbono, unidos a otros tipos de áto$\operatorname{mos}^{17}$, demuestran capacidad para formar un ilimitado número de cadenas. Este hecho hizo posible la existencia de seres vivos, pues éstos presuponen diversidad de reacciones moleculares.

La Tierra comenzó a llenarse de estas cadenas de moléculas orgánicas tanto en el aire como en los mares. Esas cadenas comenzaron a elaborar reacciones entre sí, cada vez más complejas. Estas redes crearon verdaderas familias de moléculas que, a través de las reacciones, produjeron los mismos tipos de moléculas que las integraban. Siempre que se verifica este tipo de organización, sin el cual nada existe, se dan las condiciones para la emergencia de un ser vivo. Se trata de una creación, una autopoiesis, en el lenguaje técnico de los científicos Varela y Maturana. Podríamos decir que se trata de la auto-organización de la materia. De ahí que la vida surja como consecuencia de la creciente complejización. ${ }^{18}$ En consecuencia, cuando aparecen unidades auto-organizadas (autopoiéticas), emerge la vida. Y apa-

sión sobre la vida en la Tierra disponible en www.ebiblioteca.org].

17 Cf. "Este proceso podría verse como una especie de efecto invernadero al revés, que hizo que las primeras formas de vida se enfrentaran a un problema parecido al calentamiento global, sólo que para ellas la amenaza no era el calor, sino el frío o la congelación. Creemos que esas tempranas manifestaciones de vida resolvieron el problema mediante la evolución de organismos llamados metanógenos, que todavía sobreviven en nuestros intestinos y en lugares donde no hay oxígeno. Estos «detritóforos» viven de descomponer los cuerpos de fotosintetizadores muertos y de otros organismos. Durante el proceso se libera metano $y$ dióxido de carbono en forma gaseosa." LOVELOCK, 2007, pp. 69-70.

18 " [...]The Gaia hypothesis, on the other hand, started with observations of the Earth's atmosphere and the other inorganic properties. Where life is concerned, it focuses special attention on what most people consider to be the lowest part, that represented by the microorganisms. The human species is of course a key development for Gaia, but we have appeared so late in her life that it hardly seemed appropriate to start our quest by discussing our own relationships within her..." Cf. Trad.: "La hipótesis de Gaia, por otro lado, partió de observaciones realizadas en la atmósfera, de datos sobre características inorgánicas. Allí donde de vida se trata, lo verdaderamente fundamental son los microorganismos, comúnmente colocados en el escalón más bajo. La especie humana es, desde luego, uno de los hitos claves de Gaia, pero somos una aparición tan tardía que no parecía excesivamente apropiado dar comienzo a nuestra exposición discutiendo nuestras relaciones en ella." LOVELOCK, 2013, p.116. 
rece de un modo inevitable como resultado de un largo proceso evolutivo. Surge en muchos lugares y en varios momentos, tal vez en muchos millones de años, pero siempre y cuando se ofrecen condiciones propicias para unidades auto-organizadas.

Como lo mostraron notables científicos, las condiciones favorables a la vida no son anteriores a la aparición de la vida. La propia vida en la Tierra fue creando buenas condiciones para sí misma, en el subsuelo, en el suelo y en el aire. Fue resistiendo a los obstáculos, se fue adaptando a los cambios y fue creando, con habilidad y sacrificio, la_esfera que le era adecuada, es decir una biosfera que se autorregula. El esfuerzo de adaptación fue tan inteligente y sutil, que hasta el oxígeno en estado puro, que era, inicialmente nocivo para la vida, fue convertido en su principal suministro. Mediante la respiración, el oxígeno contenido en la atmósfera se transmuta en carbono $\left(\mathrm{CO}_{2}\right)$, que la fotosíntesis ${ }^{19}$ absorbe dando origen a biomasa y nuevamente al oxígeno. La fotosíntesis permitió que la Vida sintetice su propia materia orgánica, captando directamente la energía solar.

Surgieron, en los océanos ${ }^{20}$, organismos como renacuajos y otros microorganismos; aparecieron corales, moluscos y otros animales mayores que hacen caparazones o estructuras de carbonato de calcio y magnesio. Inmensas capas de sedimentos fueron acumuladas. El desplazamiento tectónico, más tarde, elevó muchas de ellas. Como las montañas de los Alpes, monte Atlas, Los Andes y demás cordilleras, están a la vista las estratificaciones. En algunas de ellas, podemos observar millones de años de paciente trabajo de sedimentación. Uno de los espectáculos más fantásticos de este proceso es el Gran Cañón, en Arizona. Fueron así retiradas de la atmósfera grandes cantidades de gas carbónico. Pero no bastó la fijación de este gas en forma de carbonatos. Otros organismos tuvieron que colaborar con la tarea. Surgieron los primeros grandes bosques, que incluían plantas en el nivel evolutivo

${ }^{19}$ Cf. LOVELOCK, 2007, p.69.

20 "If we are lucky we may find that the vital organs in the body of Gaia are not the Lnd surfaces but in the estuaries, wet lands, and muds on the continental shelves. There, the rate of carbón burial adjusts automatically to regulate the concentration of oxygen, and essential elements are returned to the atmosphere.", Cf. Traducción; "Con suerte, quizá descubramos que los órganos vitales del cuerpo de Gaia no están en las superficies terrestres sino en los estuarios, los pantanos y en los fangos de las plataformas continentales. Es aquí donde el ritmo de enterramiento de carbono se ajusta automáticamente al oxígeno disponible y donde son devueltos a la atmósfera elementos esenciales" LOVELOCK, 2013, p.122. 
de musgos y helechos, de licopodios, cicadáceas, palmeras, y muchas formas hoy extintas, esto ocurre en el Período Carbonífero, unos trescientos millones de años atrás. Fueron depositadas gigantescas sedimentaciones de carbón mineral y lignito. En pantanos más recientes crecieron las turbas del norte de Europa, Canadá y Siberia. En Escocia, es fácil observar como hoy crecen todavía.

Este proceso lento pero gradual suscitó la hipótesis Gaia. En ella, todos los elementos -la composición fisicoquímica, los microorganismos, las plantas, los animales, los seres humanos- jamás están simplemente yuxtapuestos unos con otros, por el contrario, ellos inter-existen y co-existen. De tal modo que son interdependientes e imbricados fundando un equilibrio que solamente se encuentra en seres vivos, como un bosque o un pantano. Así se constató $^{21}$ que los porcentajes de $21 \%$ de oxígeno, de $79 \%$ de nitrógeno en la atmósfera y de 3,4\% de salinización en los océanos perduran inalterados desde hace millones de años, aunque la luminosidad del Sol haya aumentado un 30\% desde la formación de la Tierra. ${ }^{22}$

En conclusión, escribió Lovelock en su primera publicación:

Habiendo asumido su existencia, permítasenos considerar tres características principales de Gaia que podrían modificar profundamente nuestra interacción con el resto de la biosfera.

1. La propiedad más importante de Gaia es la tendencia a mantener constantes las condiciones para la totalidad de la vida terrestre. Siempre que nosotros no hayamos interferido seriamente con su estado de homeostasis, esta tendencia debe ser tan predominante ahora como lo era antes de la llegada del hombre a la escena.

2. Gaia tiene los órganos vitales en el centro, en tanto que los prescindible o redundantes se hallan principalmente en la periferia. Lo que le hacemos a nuestro planeta puede depender enormemente dónde nosotros lo hagamos. ${ }^{23}$

${ }^{21}$ Cf. LOVELOCK, 2007, Capítulo 3.

22 Cf. "[...] Quizá por ello una de las mayores crisis a las que Gaia ha tenido que enfrentarse durante su existencia, la consolidación del oxígeno como gas atmosférico dominante, no tuvo consecuencias letales. Sucedió cuando el clima del sistema solar era benigno. Al principio, hace más de tres mil millones de años, el sol era demasiado frío. Ahora es demasiado caliente.

La aparición del oxígeno fue un acontecimiento tan importante para la existencia de Gaia como la pubertad para los humanos. Impulsó la evolución de células vivas más complejas, las eucariotas, y, con el tiempo, llevó a los inmensos conglomerados de células que son las plantas y los animales." Ibidem, p.71.

${ }^{23}$ Este punto puede comprenderse en forma total si lo complementamos con otro pasaje: "De dentro afuera desde el núcleo, la Tierra está formada casi exclusivamente de roca y 
3. Las respuestas Gaiana a los cambios para lo peor debe obedecer a las reglas de la cibernética, donde el tiempo constante y la ganancia del bucle recursivo son factores importantes. Así la regulación del oxígeno tiene una constante de tiempo medida en miles de años. Tales procesos lentos dan la menor advertencia de tendencias indeseables. Cuando se comprende que todo no está bien y la acción se ejecutó, el arrastre inercial lleva las cosas a un estado peor anterior a una mejora igualmente lenta que puede situarse dentro"( 2013, p.119)..$^{24}$

Con la finalidad de considerar las semejanzas entre la posición de Plotino y la de Lovelock procederemos a comparar la proyección de la doctrina del filósofo con la teoría del científico.

\section{7- A modo de conclusión: comparación de la proyección}

Ahora bien, dando nuevamente continuidad al tema de la proyección de la doctrina del Alma Universal y la comparación con la tesis de un Planeta viviente que se autorregula a sí mismo veremos entonces que Lovelock también hace explícita esta antigua idea al sostener que:

Debemos pensar en Gaia como un sistema integral formado por partes animadas e inanimadas. El exuberante crecimiento de los seres vivos, posible gracias al sol, hace a Gaia muy poderosa, pero

metal caliente o líquido. Gaia es un delgado caparazón esférico de materia que rodea el interior incandescente; empieza allí donde las rocas de la corteza se encuentran con el magma del interior de la Tierra, a unos cientos sesenta kilómetros bajo la superficie, y se extiende otros ciento sesenta kilómetros hacia arriba, a través del océano y el aire, hasta la todavía más caliente termosfera, que linda con el espacio exterior. Incluye la biosfera y es un sistema fisiológico dinámico que ha mantenido nuestro planeta apto para la vida durante más de tres mil millones de años. Digo que Gaia es un sistema fisiológico porque parece tener el objetivo inconsciente de regular el clima y la química de forma que resulten adecuados para la vida". Ibidem, p. 37.

24 Having assumed her existence, let us consider three of Gaia's principal characteristics which could Profundly modify our interaction with the rest of the biosphere.

1. The most important property of Gaia is the tendency to keep constant conditions for all terrestrial life. Provided that we have not seriously interfered with her state of homeostasis, this tendency should be as predominant now as it was before man's arival on the scene.

2. Gaia has vital organs at the core, as well as expendable or redundant ones mainly on the periphery. What we do to our planet may depend greatly on where we do it.

3. Gaian responses to changes for the worse must obey the rules of cybernetics, where the time constant and the loop gain are important factors. Thus the regulation of oxygen has a time constant measured in thousands of years. Such slow processes give the least warning of undesirable trends. By the time it is realized that all is not well and action is taken, inertial drag will bring things to a worse state before an equally slow improvement can set in. LOVELOCK, 2000, p.119. 
este caótico y salvaje poder está constreñido por las propias limitaciones de esa entidad que se regula a sí misma en beneficio de la Tierra (2007, p.38).

La cosmovisión del científico cobra verdadero sentido holístico en el marco de un nuevo paradigma integrador o de la complejidad. Reconociendo su valioso aporte desde ese enfoque pero motivado por lo que otros científicos anteriores a él ya habían afirmado. Así lo recuerda Deléage:

Lovelock dice que la crítica más perniciosa de sus ideas es la que pretende que no hay nada nuevo en la hipótesis Gaia, que "todo esto se conocía desde hacía mucho tiempo". Pero esta crítica se basa en un fondo de verdad. Efectivamente, la hipótesis geofisiológica se inscribe en la tradición filosófica y científica de Humboldt y los románticos alemanes en el siglo XIX, y Vernadsky y Hutchinson en el siglo XX. Así pues, a Lovelock le resulta fácil recordar que la ciencia normal rechazó fuera de su campo de investigación algunos fenómenos conocidos y observados porque no encontraban ninguna explicación para ellos en el marco de los paradigmas dominantes. $(1993,271)$.

Aunque esta concepción organicista sobre el cosmos haya predominado en la filosofía antigua y esté bastante extendida en la Ciencias de la Tierra, Lovelock hace la siguiente advertencia: “[...] Todavía nos resulta ajeno el concepto de que nosotros y el resto de la vida, desde las bacterias a las ballenas, formamos parte de una entidad mucho mayor y más diversa: la Tierra viva" (2007, P.21).

Con la intención de hacer más clara la comparación entre ambos enfoque exhibimos un cuadro que compara los puntos más relevantes de cada posición:

\begin{tabular}{|l|l|}
\hline \multicolumn{1}{|c|}{ PLOTINO / Alma Universal } & \multicolumn{1}{c|}{ LOVELOCK / GAIA } \\
\hline $\begin{array}{l}\text { Cosmos vivo gracias al Alma } \\
\text { Universal. }\end{array}$ & $\begin{array}{l}\text { Planeta Tierra es un } \\
\text { megaorganismo vivo en virtud de la } \\
\text { homeostasis o autoregulación. }\end{array}$ \\
\hline $\begin{array}{l}\text { Cosmos se caracteriza por ser vivo, } \\
\text { Sabio e inteligente. }\end{array}$ & $\begin{array}{l}\text { Lovelock considera que Gaia en } \\
\text { tanto sistema viviente es } \\
\text { inteligente. }^{25}\end{array}$ \\
\hline
\end{tabular}

25 " [...] If Gaia exists, then she is without doubt intelligent in this limited sense at the least. [...] It is to be compared in intelligence with the level of the regulatory mechanisms which we would expect to find Gaia using”. LOVELOCK, 2000, p 138. Nuestra traducción: $S i$ Gaia existe, entonces, sin duda ella es inteligente en un sentido limitado por lo menos. [...] de ser comparado en inteligencia con el nivel de los mecanismos reguladores que espera- 


\begin{tabular}{|c|c|}
\hline $\begin{array}{l}\text { El Alma inferior, Alma Universal o } \\
\text { physis procede de la Hipóstasis } \\
\text { Alma reúne propiedades de la } \\
\text { región inteligible que se } \\
\text { manifiestan en el cosmos sensible. } \\
\text { Del Alma Universal, la propiedad y } \\
\text { función que debemos destacar es la } \\
\text { del gobierno del mundo sensible y } \\
\text { de los seres que en él habitan. Entre } \\
\text { otras cualidades diremos es eterna } \\
\text { y se relaciona con las almas } \\
\text { individuales, es única, es sabiduría } \\
\text { plena, que se encarga del cuidado } \\
\text { de los seres sensibles, es inmanente } \\
\text { al Universo; lo vivifica, le da } \\
\text { movimiento y organicidad. }\end{array}$ & $\begin{array}{l}\text { La singularidad de la atmósfera } \\
\text { terrestre puede tener una sola } \\
\text { explicación: la actividad bioquímica } \\
\text { de los seres vivos. }\end{array}$ \\
\hline $\begin{array}{l}\text { Plotino es el primero en extender a } \\
\text { los demás seres vivos la capacidad } \\
\text { de contemplar, considerada hasta él } \\
\text { como una capacidad perteneciente } \\
\text { solo a los seres humanos. }\end{array}$ & $\begin{array}{l}\text { Lovelock es el primero en } \\
\text { considerar que los seres vivos } \\
\text { interactúan en beneficio de la auto- } \\
\text { regulación físico-química } \\
\text { (homeostasis). }\end{array}$ \\
\hline $\begin{array}{l}\text { El Alma Universal le otorga al } \\
\text { Cosmos orden, belleza, armonía, } \\
\text { equilibrio, inteligencia, etc. }\end{array}$ & $\begin{array}{l}\text { Le adjudica a la Tierra: equilibrio } \\
\text { dinámico (homeostasis), } \\
\text { autorregulación, vida, orden, etc. }\end{array}$ \\
\hline
\end{tabular}

Respecto del cuadro podemos comparar la existencia de analogías entre la perspectiva de Plotino y la propuesta científica de Lovelock. Decimos que hay analogías entre ambos enfoques porque es imposible equiparar los supuestos de cada una de las parte. De ahí que distingamos que para Lovelock:

a) La Tierra es un gran organismo viviente con la capacidad de autoregular sus propias variables ambientales: oxígeno, gases, hume-

ríamos encontrar a Gaia usando. 
dad, salinidad de los mares, temperatura ambiental, etc. haciendo propicias las condiciones para la vida en la biosfera.

b) El universo bacteriano contribuye para que las condiciones sean benéficas para el resto de los seres vivos.

c) La Tierra es inteligente en la medida en que los procesos cibernéticos u homeostáticos son mecanismos automáticos de regulación (no habría una "conciencia" que actúe a favor de ella).

En cambio, Plotino, en una dimensión filosófica, argumenta que:

a) El cosmos; la Tierra, es un organismos vivo gracias a la presencia del Alma Universal y la Providencia.

b) No existe, por supuesto, alusión al nivel microscópico debido a que en época de Plotino ese estrato biológico era desconocido. Aunque Plotino amplía la capacidad de contemplación tanto a plantas como animales, algo semejante sucede en el caso de la $\mathrm{Ti}$ erra.

c) El Cosmos y la Tierra son inteligentes gracias a la presencia y existencia del Alma Universal y al logos manifestativo de las Esencias-formas de la hipóstasis Inteligencia.

Salvando las distancias disciplinares de cada uno, tanto Plotino como Lovelock comparten la convicción común de habitar un Planeta vivo. En segundo lugar, ambos coinciden en que el planeta y el Universo no se rigen por el azar sino por la presencia de la Providencia, como en el caso de Plotino, o por las reglas de la cibernética o sistemas integrados de autorregulación, como es la posición que asume Lovelock. Ambos pensadores, cada uno por su camino, hacen una defensa del cosmos y el Planeta. Plotino, por su parte, le otorga al Cosmos dignidad ontológica a través del Alma Universal. En cambio, James Lovelock sabe que los procesos de equilibración dinámica (Homeostasis), que mantienen estable las variables químicas como el oxígeno, la salinidad de los océanos, la temperatura terrestre; son elementos en los que él se apoya para sostener que el Planeta es un ente inteligente y vivo. 
Ahora bien, el presente capítulo es una de las líneas de ecología contemporánea sobre la que se proyecta el pensamiento cosmológico de nuestro filósofo. La otra línea de ecología con la que la cosmología plotiniana comparte algunos elementos es la Ecología Profunda que será expuesta a continuación y en la que profundizaremos otra vía de la proyección.

Recebido em 05/03/2021 e aprovado em 27/03/2021

\section{Bibliografía}

Arana Marcos, Platón. Doctrinas No Escritas. Antología. Bilbao: Servicio Editorial del País Vasco/Eusakal Herrico Unibertsitatea,1998.

García Bazán, Plotino y la mística de las tres hipóstasis. Buenos Aires: El hilo de Ariadna, 2011.

Deléage, Historia de la Ecología, Barcelona: Icaria, 1993.

Orbe. Estudiosos Valentinianos. Vol. II. Roma: Universitatis Gregorianae, 1955.

Plotino. Enéadas I-IV. Traducción española de Jesús Igal con Introducción y notas, 2 volúmenes que comprenden: Porfirio, Vida de Plotino y Eneadas.Madrid: Biblioteca Clásica Gredos, 1982-1985.

Lovelock. GAIA. A new look at life on earth. England: Oxford University Press, 2000.

, GAIA, Una nueva visión sobre la vida en la Tierra. Inglaterra: Oxford University Press, 2013. Versión Word en castellano disponible en www.ebiblioteca.org.

Planeta, 2007.

La venganza de la Tierra. Buenos Aires: Editorial

Padrón. Hector Jorge, "Naturaleza, Naturalismo y Ecología". En Massini Correa, Carlos. Ecología y Filosofía. Mendoza, Argentina: Edium, 1993. Pp.131-150

Platón. Timeo. Traducción Conrado Eggers Lan. Buenos Aires: Colihue, 2005.

Zamora. La génesis de lo múltiple. Materia y mundo sensible en Plotino. Valladolid: Secretariado de Publicaciones e Intercambio Editorial de la Universidad de Valladolid, 2000. 